\title{
COMMUNICATION
}

\section{Angine bulleuse hémorragique : revue systématique de la littérature.}

\author{
Ordioni $\mathrm{U}^{1,2}$, Lan $\mathrm{R}^{2}$, Roche-Poggi P2, Campana F1, Catherine $\mathrm{JH}^{2}$
}

1. Centre Massilien de la Face - Marseille

2. Service d'Odontologie, Département de Chirurgie Orale - CHU Timone - Marseille

\section{Introduction}

L'angine bulleuse hémorragique $(\mathrm{ABH})$ a été décrite en 1967 par Badham et al. comme une pathologie entrainant « des bulles hémorragiques récidivantes de la muqueuse oropharyngée, sur des sites particulièrement exposés aux traumatismes »(1). Depuis de nombreux cas cliniques ont été décrit dans la littérature. L'objectif de ce travail était de réaliser une revue systématique de la littérature.

\section{Matériels et méthodes}

40 articles ont été sélectionnés selon la méthode PRISMA et ont permis de référencer 225 cas de AHB. Les caractéristiques épidémiologiques, étiologiques, cliniques, histologiques, biologiques et thérapeutiques ont été répertoriées.

\section{Résultats}

L'âge moyen des patient était de 55,4 ans, le sex-ratio de 0,7. La localisation préférentielle était le palais $(65 \%)$. Deux tiers des patients ne présentaient aucun antécédent médical. Un facteur favorisant existait dans $82 \%$ des cas, les lésions apparaissaient le plus souvent au décours de l'alimentation (64\%). Le diagnostic était clinique et paraclinique. La numération plaquettaire et le bilan de la coagulation, respectivement prescrit chez $63 \%$ et $43 \%$ des patients étaient toujours négatifs. Une biopsie était réalisée chez $43 \%$ des patients, la moitié avec une IFD, cette dernière étant toujours négative. Le traitement était symptomatique. L'évolution était la plupart du temps favorable. Un drainage de la bulle était réalisé chez certains patients afin de limiter son évolution ; Cette dernière pouvant obstruer les voies aéro-digestives supérieures (2). Les récidives étaient fréquentes $(65 \%)$.

\section{Discussion}

L'ABH est une pathologie peu connue. Elle n'est pourtant pas rare. La localisation est buccale dans $20 \%$ des cas rendant le terme d'angine bulleuse hémorragique inapproprié, le terme de stomatite bulleuse hémorragique bénigne proposé par Antoni-Bach et al. pourrai être plus adapté (3). L'étiologie est controversée, cette revue ne permet aucune association étio-pathogénique avec une pathologie générale ou un traitement médicamenteux comme cela a été précédemment décrit dans la littérature (utilisation de corticoïde en inhalation, diabète, hypertension). Les diagnostics différentiels sont nombreux et les moyens diagnostiques varient en fonction des auteurs. L'établissement de critères diagnostics pourrait être utile et permettrait de standardiser ce dernier.

ugo.ordioni@gmail.com

\section{Références}

1. Badham NJ. Blood blisters and the oesophageal cast. J Laryngol Otol. juill 1967;81(7):791-803.

2. Pahl C, Yarrow S, Steventon N, Saeed NR, Dyar O. Angina bullosa haemorrhagica presenting as acute upper airway obstruction. Br J Anaesth. févr 2004;92(2):283-6.

3. Antoni-Bach N, Couilliet D, Garnier J, Tortel MC, Grange F, Guillaume JC. [Case for diagnosis. Benign hemorrhagic bullous stomatitis]. Ann Dermatol Vénéréologie. juill 1999;126(6-7):525-6.

(C) The authors, published by EDP Sciences. This is an Open Access article distributed under the terms of the Creative Commons Attribution License 4.0 (http://creativecommons.org/licenses/by/4.0/). 